\title{
Optimization of oxygen transfer through venturi-based systems applied to the
}

\section{biological sweetening of biogas}

Ginesta Rodriguez*, Antonio David Dorado*, Anna Bonsfills *, Ricard Sanahuja*, David Gabriel*** and Xavier Gamisans*

*Universitat Politècnica de Catalunya, Bases de Manresa 61-73, 08240 - Manresa, Spain

** Universitat Autònoma de Barcelona, Edifici C, 08193 - Bellaterra, Spain

Correspondence: Xavier Gamisans (xavierg@emrn.upc.edu), EPSEM, Universitat Politécnica de Catalunya (UPC). Bases de Manresa, 61-73, 08242 Manresa, Spain. Tel. $+34938777324$

\footnotetext{
Abstract:

BACKGROUND: Dissolved oxygen is a key parameter in the biological removal of gaseous $\mathrm{H}_{2} \mathrm{~S}$ since sulfide-oxidizing bacteria convert $\mathrm{H}_{2} \mathrm{~S}$ into elemental sulfur instead of sulfate under limiting dissolved oxygen concentrations. The elemental sulfur is insoluble in water and accumulates in the packing material in biotrickling filters, increasing the pressure drop and operating costs. A set of mass transfer tests were performed in a versatile pilot plant to supply the necessary oxygen for the biological oxidation of $\mathrm{H}_{2} \mathrm{~S}$. The plant can be operated with three different commercial gas-liquid contactor devices: a venturi ejector, a jet-venturi and a diffuser. The effects of different geometric and operating parameters have been tested for each device (including pressure rise and sulfate content to mimic real waste water treatment plant conditions).

RESULTS: Results showed that the bioreactor pressure has a strong influence on the mass transfer characteristics of the three oxygenation devices. In addition, the ionic strength of the solution exerts a low influence on the mass transfer process.
} 
The SOTE values obtained for the diffuser, jet-venturi and venturi ejector were $8.7 \%$, $7.2 \%$ and $6.0 \%$ respectively (pure water experiments), while SAE values were in the range $6.3-5.5 \cdot 10^{-2}, 4.1-4.8 \cdot 10^{-1}$ and $3.1-3.7 \cdot 10^{-1} \mathrm{~kg} \mathrm{O}_{2} \mathrm{~kW}^{-1} \mathrm{~h}^{-1}$, respectively.

CONCLUSIONS: The jet-venturi offers the best oxygen transfer with lower power consumption compared to the diffuser and venturi ejector. The practical implementation of the most efficient aeration technology is possible from both technical and economical perspectives

Key words: Mass transfer, biogas desulfurization, biotrickling filters, jet-venturi, venturi ejector, diffuser.

\section{Nomenclature:}

$\mathrm{C}_{\text {Lin }}=$ Dissolved oxygen concentration at the inlet of the column and the outlet at the tank, $\mathrm{kg} \mathrm{m}^{-3}$

$\mathrm{C}_{\text {Lout }}=$ Dissolved oxygen concentration at the outlet of the column, $\mathrm{kg} \mathrm{m}^{-3}$

$\mathrm{C}_{\mathrm{S}}=$ Dissolved oxygen concentration at saturation, $\mathrm{kg} \mathrm{m}^{-3}$

$\mathrm{K}=(\gamma-1) / \gamma=0.283$ for air with adiabatic compression

$\gamma=$ Ratio of specific heat for gas $=1.395$ for air with adiabatic compression

$\mathrm{K}_{\mathrm{L}} \mathrm{a}=$ Mass transfer coefficient, $\mathrm{s}^{-1}$

$\mathrm{K}_{\mathrm{L}} \mathrm{a}_{20}=$ Mass transfer coefficient corrected at $20^{\circ} \mathrm{C}$ and pressure of $1 \mathrm{~atm}, \mathrm{~s}^{-1}$

$\mathrm{P}_{1}=$ Absolute pressure before compression, $\mathrm{kPa}$

$\mathrm{P}_{2}=$ Absolute pressure after compression, $\mathrm{kPa}$

$\mathrm{P}_{\text {In }}=$ Power input, $\mathrm{kW}$

$\mathrm{Q}_{\mathrm{G}}=$ Gas flowrate, $\mathrm{m}^{3} \mathrm{~s}^{-1}$

$\mathrm{Q}_{\mathrm{L}}=$ Recirculation liquid flowrate, $\mathrm{m}^{3} \mathrm{~s}^{-1}$

$\mathrm{R}=\mathrm{Gas}$ constant for air $=2.89 \cdot 10^{-3} \mathrm{~kW} \mathrm{~s} \mathrm{~g}^{-1} \mathrm{~K}^{-1}$

$\mathrm{SAE}=$ Standard Aeration Efficiency, $\mathrm{Kg} \mathrm{O}_{2} \mathrm{~kW}^{-1} \mathrm{~h}^{-1}$ 
$\mathrm{SG}=$ Specific gravity of water at the test temperature

SOTE $=$ Standard Oxygen Transfer Efficency, dimensionless

SOTR =Standard Oxygen Transfer Rate, $\mathrm{Kg} \mathrm{O}_{2} \mathrm{~h}^{-1}$

$\mathrm{t}=$ experimental time, $\mathrm{s}$

$\mathrm{T}_{1}=$ Absolute temperature before compression, $\mathrm{K}$

$\mathrm{TDH}=$ Total dynamic head, $\mathrm{m}$

$\mathrm{TLV}=$ trickling liquid velocity, $\mathrm{m} \mathrm{h}^{-1}$

$\mathrm{V}_{\mathrm{LC}}=$ Volume of the column, $\mathrm{m}^{3}$

$\mathrm{V}_{\mathrm{T}}=$ Volume of the tank, $\mathrm{m}^{3}$

$\mathrm{w}=$ Mass flow of gas, $\mathrm{g} \mathrm{s}^{-1}$

$\mathrm{W}=$ Specific weight of water at test temperature, $\mathrm{N} \mathrm{m}^{-3}$

$\mathrm{W}_{\mathrm{O} 2}=$ Mass flow of oxygen in the air stream, $\mathrm{Kg} \mathrm{O}_{2} \mathrm{~h}^{-1}$

\section{Introduction}

Biofiltration has proven to be a good alternative to physical-chemical systems for the removal of hydrogen sulfide $\left(\mathrm{H}_{2} \mathrm{~S}\right)$ present in energy-rich gases such as biogas ${ }^{1}$. In biofilters $(\mathrm{BF})$ or biotrickling filters $(\mathrm{BTF})$ the dissolved oxygen concentration is a critical parameter for the biological degradation of $\mathrm{H}_{2} \mathrm{~S}$. The amount of oxygen transferred to the aqueous phase is directly responsible of the formation of sulfate or elemental sulfur according to eqs. 1 and $2^{2}$.

(1) $\mathrm{HS}^{-}+0.5 \mathrm{O}_{2} \rightarrow \mathrm{S}^{0}+\mathrm{OH}^{-}$

(2) $\mathrm{HS}^{-}+2 \mathrm{O}_{2} \rightarrow \mathrm{SO}_{4}{ }^{2-}+\mathrm{H}^{+}$

Aqueous sulfate is removed from the biofilter through the renewal of the liquid phase, while elemental sulfur, which is insoluble in water, is mostly retained in the packing material. Elemental sulfur accumulation has been observed in BTFs operating at highloads of $\mathrm{H}_{2} \mathrm{~S}$ (>100 $\left.\mathrm{g} \mathrm{H}_{2} \mathrm{~S} \mathrm{~m}^{-3} \mathrm{~h}^{-1}\right)^{3}$ leading to increasing pressure drop and increasing 
operating costs for the equipment. Eventually, elemental sulfur accumulation can lead to the complete clogging of the bed.

This phenomenon has been observed in a full-scale BTF located at the waste water treatment plant (WWTP) of Manresa. From the analysis of mass balances performed during the steady operation of the plant, an accumulation of $2.35 \mathrm{~kg} \mathrm{~S}^{0}$ day $^{-1}$ was detected when treating $\mathrm{H}_{2} \mathrm{~S}$ concentrations up to $3000 \mathrm{ppm}_{\mathrm{V}}{ }^{4}$. From this perspective, an improvement of the oxygen supply to the system is mandatory and should be performed without significant changes in the biogas quality as fuel. A common practice at industrial scale in most of the biogas treating BTF is to supply oxygen (or air) mixed with the $\mathrm{H}_{2} \mathrm{~S}$ loaded stream, so almost no improvement can be made with this configuration. Due to the low solubility of oxygen in comparison with $\mathrm{H}_{2} \mathrm{~S}$ a dissolved oxygen deficit is usually found in these configurations.

On the other hand, venturi-based devices have already demonstrated to offer high mass transfer rates when used as gas-liquid contactors in scrubbing facilities ${ }^{5}$. Gas-liquid mass transfer characterization is performed by calculating global (or local) mass transfer coefficients based on mass balances formulated both in the gas and in the liquid phase in the $\mathrm{BTF}^{6}$. Other methodologies include the use of a liquid phase reaction (e.g. sulfite oxidation) for local determinations ${ }^{7}$.

Optimization of oxygen mass transfer is, thus, a priority objective in order to widen the application of biological technologies to energy-rich gas desulfurization. This optimization step lies basically on the search of intensive gas-liquid contactors suitable to be included in BTF designs. According to the stated above, the aim of the present study was to optimize the oxygen transfer from air into water or aqueous solutions by simulating BTF liquid phase conditions. In this sense, three commercially available 
intensive mass transfer devices were used for comparison purposes (a jet-venturi, a venturi ejector and a membrane diffuser).

\section{Experimental}

Figure 1 shows a schematic of the pilot plant used for the mass transfer tests. It consists of a 2001 tank (SV) equipped with one downward tube (DT) were the venturi-based devices are placed. The liquid phase is recirculated with a centrifugal pump (PFV) (Model CPS, Nocchi, Spain) from the bottom of the tank to the top of the DT. Dissolved oxygen concentration (DO) was measured (CellOx 325, WTW, Spain) at two points, corresponding to the outlet of the tank (DO1), and at the end of the DT (DO2). Three different gas-liquid contactor devices were used: a venturi ejector (Models 878-2, 885X2 and 978-2, Copersa, Spain), a jet-venturi (Model 2, Tecnium, Spain) and a membrane diffuser (Roeflex-roediger, Sacede, Spain). A previous series of experiments were performed to determine the most efficient (in terms of entrained air flow) venturi ejector model. Main geometric differences between models are related to the throat and diffuser section dimensions which give different throat section volumes: $1.76 \mathrm{~cm}^{3}$ for $878-2$ model, $2.34 \mathrm{~cm}^{3}$ for $885 \mathrm{X}-2$ model and $1.83 \mathrm{~cm}^{3}$ for $978-2$ model.

The centrifugal pump was equipped with a frequency variator. The recirculated liquid was measured with a flow meter (FI3) (Model 5L61, Key Instruments, Canada) and a hydraulic shock absorber (EV) was also placed to keep a stable pressure during the experiments. The short loop circuit in Figure 1 was used during the diffuser-based experiments (for DO measurements purpose). Entrained air (secondary fluid) for the ejector and the jet-venturi was measured with a flow meter (Model FR400, Key Instruments, USA) (FI1). Compressed air supplied to the diffuser was also measured with a flow meter (Model PT-313-0400, Tecfluid, Spain) (FI2). Prior to each experiment, and in order to deoxygenate the system, the aqueous phase was sparged 
with nitrogen from a cylinder (99.99\%, Abelló Linde, Spain). Initial DO concentration was kept below $3 \mathrm{mg} \mathrm{l}^{-1}$ in each test.

Different system pressures were tested in the range of 0.1 to 0.5 bar, corresponding to usual biogas storage pressures in WWTP ${ }^{4}$. In order to obtain comparable results, the air flowrate was maintained at $201 \mathrm{~min}^{-1}$ in all experiments. Thus, the liquid flowrate was increased as the pressure inside the tank rose to supply the same amount of air to the system. This allowed to check the effect of pressure changes in the amount of oxygen supplied to a BTF as well as to assess its positive (increase in oxygen solubility) and negative (loss of suction capacity) effects. A high-precision digital pressure switch (ZSE40/ISE40, SMC, Technical, Spain) connected to a safety valve (PSV) (Technical, Spain) was used to maintain stable pressure values inside the tank.

The effect of the ionic species content on the oxygen mass transfer was studied by using different aqueous sulfate concentrations ranging from 0 to $1000 \mathrm{mg}^{-1}$. The impact of ionic species concentration is also a critical parameter that may provide useful information regarding the suitable aqueous phase renewal in the BTF from an optimal oxygen supply perspective. Despite sulfate concentrations tested are far from $1.9 \mathrm{~g} \mathrm{SO}_{4}$ $\mathrm{l}^{-1}$, that is the inhibitory concentration reported in biotrickling reactors ${ }^{8}$, concentrations tested were representative of those found in real applications. In the present work, sulfate concentrations tested were selected based on concentrations found under steady operation of the full-scale (industrial) BTF at the Manresa WWTP. In this case, water renewal rate is used for $\mathrm{pH}$ control ${ }^{4}$, which leads to low (between 500-600 $\mathrm{mg} \mathrm{l}^{-1}$ ) sulfate concentrations in the liquid phase. Sulfate concentration was determined by ion chromatography with conductivity detection in a DIONEX ICS-1000 (Dionex, Spain). According to literature, the sensor response time must be less or equal to $1 / \mathrm{K}_{\mathrm{L}} \mathrm{a}$ in order to neglect the dynamics of the oxygen probe in the calculations of mass transfer 
coefficients ${ }^{9}$. The method from Vandu and Krishna (2004) ${ }^{10}$ was used for the determination of the sensor response time. The test indicated that the effect of sensor response time was negligible in this study.

Three replicates of the basic experiments were carried out to determine the experimental error in the determination of mass transfer coefficient. Calculated errors were lower than $10 \% \mathrm{~s}$ which is less than the $15 \%$ from ASCE recommendations ${ }^{11}$.

\section{Data acquisition}

A home-made SCADA system was built in LabWindows ${ }^{\mathrm{TM}}$ for data acquisition. Dissolved oxygen signals from electrodes (Cellox 325, WTW, Spain) were acquired using either an Inolab740 (WTW, Spain) terminal and an Oxi 340 (WTW, Spain) terminal. Communication between devices and the monitoring computer was performed via $\mathrm{RS}-232$.

\section{Calculation of the liquid-side volumetric mass transfer coefficient}

The dynamic method was used for the determination of the overall mass transfer coefficient $\mathrm{K}_{\mathrm{L}} \mathrm{a}\left(\mathrm{s}^{-1}\right)$. The method was adapted to the experimental conditions and to the pilot plant characteristics. To apply the methodology some additional assumptions were needed ${ }^{12}$. The most relevant lies on the fact that the tank and the DT were considered continuous stirred tank reactors (CSTR). Also it was assumed that mass transfer only takes place in the ejector/jet-venturi and in DT. Therefore, the equations used for quantification of the mass transfer coefficient are the following ( 3 and 4):

For the column:

(3) $V_{L C} \cdot \frac{d C_{\text {Lout }}}{d t}=V_{L C} \cdot K_{L} a \cdot\left(C_{S}-C_{\text {Lout }}\right)$

For the tank:

(4) $\frac{d C_{\text {Lin }}}{d t}=\frac{Q_{L}}{V_{T}} \cdot\left(C_{\text {Lout }}-C_{\text {Lin }}\right)$ 
Unlike most of the works found in literature related to oxygen mass transfer in venturibased equipment, in the present work equations 3-4 were solved simultaneously using a Nelder-Mead simplex direct search algorithm programmed with MATLAB. This approach allowed including the oxygen inlet concentration as process variable. This is a more realistic approach than those considering a constant inlet concentration ${ }^{13,14,15}$. This is, thus, a more generalist approach which also avoids using particular boundary conditions ${ }^{12}$.

\section{Additional calculations}

Parameters defined according to ASCE/EWRI 2-06 were utilized ${ }^{11}$ for comparison purposes of the intensive gas-liquid mass transfer devices:

The Standard Oxygen Transfer Rate (SOTR, $\mathrm{Kg} \mathrm{O}_{2} \mathrm{~h}^{-1}$ ), (Eq. 5) was used to compare the amount of oxygen transferred to the aqueous phase:

(5) $\operatorname{SOTR}=K_{L} a_{20} \cdot C_{S} \cdot V_{T}$

The Standard Aeration Efficiency ( $\mathrm{SAE}, \mathrm{Kg} \mathrm{O}_{2} \mathrm{~kW}^{-1} \mathrm{~h}^{-1}$ ) allows considering the power supplied to the device (equation 6). This is a more objective comparison criterion since it includes energetic (i.e. economical) aspects.

(6) $S A E=\frac{S O T R}{P_{I n}}$

Where the power input was calculated with the following equations:

(7) $P_{\text {In Compressor }}=\frac{w \cdot R \cdot T_{1}}{K}\left[\left(\frac{P_{2}}{P_{1}}\right)^{K}-1\right]$

(8) $P_{\text {In Water pump }}=Q_{L} \cdot T D H \cdot W$

In Eq. 7, the delivered blower power (used for the diffuser characterization) must be calculated based on adiabatic compression. Eq. 8 is the delivered pump power used in the calculations for the jet-venturi and venturi-ejector. Another parameter which provides useful comparison criteria of devices is the Standard Oxygen Transfer Efficiency (SOTE, dimensionless) (Eq. 9): 
(9) $S O T E=\frac{\text { SOTR }}{W_{O_{2}}}$

\section{Results}

In order to evaluate the individual mass transfer coefficient for each operating condition, the inlet and the outlet oxygen concentrations were recorded and adjusted to the mathematical model described above. As an example of the methodology used, Figures $2 \mathrm{a}$ and $2 \mathrm{~b}$ show a comparison between experimental and simulated values for two specific tests. The $\mathrm{K}_{\mathrm{L}} \mathrm{a}_{20}$ obtained was $23.8 \mathrm{~h}^{-1}$ (figure $2 \mathrm{a}$ ) for the venturi ejector and $32.6 \mathrm{~h}^{-1}$ (figure $2 \mathrm{~b}$ ) for the jet-venturi. As observed, the simple dynamic model described by equations 3 and 4 was perfectly able to predict the experimental behavior. This confirms the suitability of the CSTR assumption made for the venturi-based devices. This is probably due to the short contact time between the gas and liquid phases (below $0.5 \mathrm{~s}$ ).

\section{Preliminary tests}

In the evaluation of mass transfer with the three venturi ejectors, model 978-2 offered the lowest aspirated gas flow compared with the other models. The model 978-2 has a total volume in the throat higher than $20 \%$ with respect to the others ejectors.

Models 878-2 and 885X-2 offered similar $\mathrm{K}_{\mathrm{L}} \mathrm{a}_{20}$. However, the entrained air flow values were larger for model $885 \mathrm{X}-2$. For this reason, model $885 \mathrm{X}-2$ was used in the rest of experiments to compare with the diffuser and the jet-venturi.

$\mathrm{K}_{\mathrm{L}} \mathrm{a}_{20}$ values determined for the basic experiments (pure water and atmospheric pressure) were in the range of $21-38 \mathrm{~h}^{-1}$ for the diffuser, $19-33 \mathrm{~h}^{-1}$ for the jet-venturi and 15-24 $\mathrm{h}^{-1}$ for the venturi ejector. Apparently the diffuser supplies oxygen into the water phase more efficiently than the other devices. However, the interfacial area (a) for this device is considerably higher than those on venturi-based devices. For the diffuser experiments, mass transfer takes place in the whole volume of the tank, while only the 
downward tube is mass-transfer active for the venturi-based devices. Therefore the interfacial area considered with diffuser is higher when compared to venturi-based devices. $\mathrm{K}_{\mathrm{L}}$ a values found in literature were $3.6-180 \mathrm{~h}^{-1}$ for a perforate plate (similar to diffuser) ${ }^{16}, 360-1800 \mathrm{~h}^{-1}$ for a jet-venturi ${ }^{17}$ and $54-108 \mathrm{~h}^{-1}$ for a venturi ejector ${ }^{18}$. Despite literature $\mathrm{K}_{\mathrm{L}} \mathrm{a}$ are higher than those in the present study, the differences in the mass transfer equipment dimensions hinder any comparison.

In order to comparatively assess the capabilities of each mass transfer device, the amount of oxygen supplied to the water phase was related to the power consumption. In this sense, the SAE parameter is the most suitable indicator of the operating cost of the different oxygen transfer systems (including the current system used in the industrial BTF).

Figure 3 shows the SAE values for the three mass transfer devices at three different entrained (or supplied in the case of the diffuser) gas flows. The venturi-based equipment provided the highest SAE, in other words, it offers the best ratio between the oxygen transferred and the power consumed. Besides, the ejector and the jet-venturi are quite sensitive to the supplied energy still allowing further improvements at reasonably low energy requirements. It can also be seen that the jet-venturi offered a considerably higher oxygen transfer under the same operating conditions. This phenomenon was related to the appearance of different flow patterns (bubbly or annular-jet). Flow patterns were determined by visual observation and its effect on mass transfer must be further investigated. In this sense, the presence of bubbly flow might offer a higher interfacial area than annular flow ${ }^{19}$. However, the entrained air-flow is considerably increased when the two-phase flow after the venturi throat shows annular-type patterns 20 
The system efficiency can also be assessed by using the SOTE \%, that is the amount of oxygen transferred from the oxygen supplied. Values obtained where considerably low $(9 \pm 0.9 \%$ for the diffuser, $8 \pm 0.8 \%$ for the jet-venturi and $7 \pm 0.7 \%$ for the venturi ejector). However these values might be high enough to supply the necessary amount of oxygen in a BTF by appropriately installing the mass transfer equipment in the recirculation line. In addition, these values must be considered as guidance since a process scale-up should be performed previous to the final application at an industrial BTF.

In summary, the diffuser showed the best SOTR and SOTE even though the jet-venturi is the most suitable equipment in terms of power efficiency related with the amount of oxygen supplied. Besides, the diffuser membrane is susceptible to clogging with biomass or elemental sulfur particles.

In order to check the theoretical adequacy of the tested equipment, the SOTE was evaluated in the current industrial BTF in which the aeration system consists of blower that discharges air into a submerged open pipe located at the bottom of the BTF. A $\mathrm{K}_{\mathrm{L}} \mathrm{a}_{20}$ of $3.8 \mathrm{~h}^{-1}$ was found for such system, corresponding to a SOTE of $1.44 \%$, which is only $20 \%$ of the SOTE obtained with the jet-venturi. Therefore, an improvement in the BTF desulfurization efficiency as well as a positive economical impact will be obtained by replacing the existing blower by a jet-venturi equipment. This will contribute to a general improvement from an economical point of view of the biogas treatment technology based on biological systems.

\section{Effect of system pressure on the oxygen mass transfer}

Figure 4 confirms the improvement of mass transfer when the pressure inside the mass transfer equipment is increased. This improvement is related to the oxygen solubility increase in the aqueous phase and, thus, to the increase on the mass transfer driving 
force. SOTR enhancement was obtained at the expense of some extra power consumption. The hydrodynamic behavior of the jet-venturi was again responsible of its better results when compared to the venturi ejector. SAEs obtained in these tests were lower than those obtained at atmospheric pressure. Conversely, SOTEs were significantly superior, with values up to $16 \%$ for the experiments with the jet-venturi at the highest pressure tested. Although SAEs were low under relatively high pressure (in the industrial BTF the usual working pressure ranges from 0.45 to 0.5 bars), SOTEs indicate that the high energy consumption is compensated with a large amount of oxygen transferred to the system ${ }^{21}$.

\section{Effect of the sulfate content on the oxygen transfer}

Figure 5(a) demonstrates that sulfate concentration did not strongly affect mass transfer in the ejector in the range tested. Tobajas and García-Calvo $(2000)^{22}$, concluded that the salts content does not have any effect on mass transfer in experiments performed with different concentrations of glucose in a fermentation medium. However, Painmanakul and Hérbard (2008) ${ }^{23}$ showed that the mass transfer coefficient increased for a $\mathrm{NaCl}$ content of the water phase between 4000 and $20000 \mathrm{mg} \mathrm{l}^{-1}$, even if the interfacial area (a) was more affected than the liquid-side mass transfer coefficient $\left(\mathrm{k}_{\mathrm{L}}\right)$ with the increase of salts content. The moderately low sulfate concentrations used in this study (according to real conditions) was responsible of the low influence of this parameter on the mass transfer.

\section{Preliminary calculations for the implementation in an industrial BTF}

The current oxygen supply system at the industrial BTF at the Manresa WWTP consists of a blower which delivers air to the sump of the reactor. Under the present operating conditions the aeration system offers a SOTE of $1.44 \%$, which is only a $20 \%$ out of the SOTE obtained with the intensive gas-liquid contactors under study. According to this, 
replacement of the existing system for a venturi-based option is warranted. An increase in the SOTE would favorably impact on the complete oxidation of $\mathrm{H}_{2} \mathrm{~S}$ to sulfate, avoiding the accumulation of sulfur particles in the packing material. This would allow a longer operating time without unscheduled shut-downs for sulfur withdrawal as well as to reduce the operating costs related to the pressure drop through the packed bed. Other benefits coming out from the plant modifications are related to the lower electrical power consumption since the blower would be no longer needed.

To install a venturi-based device on the full-scale BTF some changes are needed and some questions need to be evaluated based on the results found herein. The following discussion is related to the logical steps needed for the practical implementation of the best available technology for the industrial BTF.

Using the existing centrifugal pump, which pumps the recycling liquid from the sump of the reactor to the irrigation system on top of the packed bed, two configurations are possible for both the jet-venturi and the venturi ejector (See supplementary material). The simplest way to integrate the gas-liquid contactor in the BTF is by installing it on the recirculation line and using the centrifugal pump delivered power to both feed the sprinklers and provide primary energy to the venturi device for air entrainment. The only limitation of this configuration lies on the relatively high liquid flowrates needed to supply enough oxygen for the highest $\mathrm{H}_{2} \mathrm{~S}$ loads. This would require the operation at high trickling liquid velocities (TLV). In this sense, an upper limit of $20 \mathrm{~m} \mathrm{~h}^{-1}$ is recognized as the maximum TLV for most BTF applications due to possible biomass detachment ${ }^{24}$. In addition, the flooding effects could be another important limitation depending on the bed porosity and packing material characteristics. In order to overcome this limitation and, thus, extent the applicability of the mass transfer equipment tested in this study, another configuration which requires minimum changes 
in the industrial BTF is possible. This configuration consists of adding a T-junction at the outlet of the venturi-based device to derive the sprinklers feeding line from an internal loop used to recirculate most of the liquid flowrate used for air entrainment. This configuration would allow a better control of both the motive fluid for air entrainment and the TLV according to each operating situation.

According to the SOTR values obtained in this study, and using the stoichiometrically necessary oxygen for the complete biological oxidation of $\mathrm{H}_{2} \mathrm{~S}$ to sulfate, a theoretical comparison of the suitability of each venturi-based device has been performed considering the two configurations described above. The test has been done by assuming an average biogas flowrate fed to the BTF of $80 \mathrm{~m}^{3} \mathrm{~h}^{-1}$ (present value) and $\mathrm{H}_{2} \mathrm{~S}$ concentrations from 500 to $3000 \mathrm{ppm}_{\mathrm{V}}$ (corresponding to the concentrations range recorded in a 12 months period). Table 1 shows the theoretical performance of the jetventuri and the venturi ejector for the first configuration (insertion of the mass transfer enhancement device directly in the recirculation line). As can be observed, the venturi ejector fails to feed enough air with a reasonable TLV. Less than $1500 \mathrm{ppm}_{\mathrm{V}}$ can be treated without surpassing the $20 \mathrm{~m} \mathrm{~h}^{-1} \mathrm{TLV}$ limitation. Conversely, the jet-venturi option may entrain oxygen enough for the successful operation of the full-scale BTF. The situation is similar when using the second configuration (T-junction to split the trickling flow and the recirculation from the venturi). In this sense, table 2 shows the gas and liquid flowrates for the same operating conditions as in table 1. In this scenario, the TLV is not a critical parameter because the loop for air entrainment and the recirculation to the sprinklers can be operated independently. Therefore both venturibased devices could be installed to satisfy the industrial BTF requirements in terms of oxygen supply. Obviously the jet-venturi would be the best available technology due to the lower energy requirements (lower liquid flowrate) for the same operating conditions. 
Despite the venturi ejector is regarded as a better gas-liquid mixer (due to the appearance of bubbly flow) the physical absorption of oxygen is governed mainly by the amount of air entrained to the contactor. Regarding to the upgrading costs of the industrial BTF, the main investment cost is related to the substitution of the current centrifugal pump (10 hp, supplying $10 \mathrm{~m}^{3} \mathrm{~h}^{-1)}$ by a higher capacity pump. Piping works will be reduced just to the installation of a T-junction and a flowmeter.

For the practical implementation of venturi devices into a BTF, the primary disadvantage is that a large flow of water must be pumped into the sump of the BTF, leading to a reduced settling capacity of the elemental sulfur accumulated in the bottom of the sump due to turbulence. Under these conditions, the drainage of elemental sulfur from the reactor would be hindered. In addition, mechanical pumping could probably warm the water phase. In this sense, a temperature increase is helpful for mesophilic microorganisms but diminishes dissolved oxygen solubility.

The addition of high amounts of air into the BTF must be performed always taking into account the explosive threshold (between 5-15\% volume of methane into air). Theoretical calculated conditions for the industrial BTF application are far from these values. From a practical application point of view, only a readjustment of the combined heat and power (CHP) plant burner is needed.

\section{Conclusions}

Preliminary tests of the oxygen transfer rate improvement in BTFs have been performed in a versatile pilot plant. Despite membrane diffusers could offer better SOTR and SOTE (i.e. the quantity of oxygen transferred and \% of oxygen transferred to the amount of air supplied) than the jet-venturi and venturi ejector, the diffuser gave the worst efficiency in energetic terms. Besides, diffuser is not suitable to be used in BFT 
because of the relatively high suspended solids concentration in the liquid phase, with the consequent clogging issues. An increase on the system pressure improves the mass transfer by means of an increase of the driving force. Conversely, the ionic strength of the aqueous phase does not considerably affect the amount of oxygen transferred even though the conditions tested were not extremely severe. Preliminary calculations showed that the venturi-based aeration systems could be a technical and economical favorable alternative in BTF devoted to energy-rich gases desulfurization.

\section{Acknowledgements:}

This work was financially supported by the Spanish Comisión Interministerial de Ciencia y Tecnologia (CICYT) project CTM2009-14338-C03-03. The authors gratefully acknowledge Javier Muñoz and Annabel Reyes for their help in the lab tests.

\section{References:}

1. Kim $\mathrm{S}$ and Deshusses $\mathrm{MA}$, Understanding the limits of $\mathrm{H}_{2} \mathrm{~S}$ degrading biotrickling filters using a differential biotrickling filter. Che Eng J 113(2-3):119-126 (2005).

2. Madigan MT, Martinko JM and Parker J, Brock Biology of microorganisms, ed by Benjamin Cummings,12th ed. Pearson education, San Francisco, p. 598 (2009).

3. Fortuny M, Baeza JA, Deshusses MA, Gamisans X, Casas C, Lafuente J and Gabriel D, Biological sweetening of energy gases mimics in biotrickling filters. Chemosphere 71:10-17 (2008).

4. Tomàs M, Fortuny M, Gamisans X, Bonsfills A, Gabriel D and Lafuente J, Design and operation of a full-scale biotrikling filter for $\mathrm{H}_{2} \mathrm{~S}$ removal from biogas, Biotechniques for Air Pollution Control, La Coruña, (2007).

5. Gamisans X, Sarrà M; Lafuente FJ, Gas pollutants removal in a single and two-stage ejector-venturi scrubber. J Hazard Mater 90:251- 266 (2002). 
6. Dorado AD, Rodríguez G, Ribera G, Bonsfills A, Gabriel D, Lafuente J and Gamisans X, Evaluation of mass transfer coefficients in biotrickling filters: experimental determination and comparison to correlations. Che Eng Tech 32(12):19411950 (2009).

7. Cooper C, Fernstrom G and Miller S, Performance of agitated gas-liquid contactors. Industrial and Engineering Chemistry 36:504-509 (1944).

8. Jin Y, Veiga M and Kennes M, Autotrophic deodorization of hydrogen sulfide in a biotrickling filter. J Chem Technol Biot 80(9): 998-1004 (2005)

9. Van't Riet K, Review of measuring methods and results in nonviscous gas-liquid mass transfer in stirred vessels. Industrial and Engineering Chemistry Process Design and Development 18:357-364 (1979).

10. Vandu CO and Krishna R, Volumetric mass transfer coefficients in slurry bubble columns operating in the churn-turbulent flow regime. Chem Eng Process 43:987-995 (2004)

11. ASCE/EWRI 2-06 Measurement of Oxygen Transfer in Clean Water, ed. ASCE. United States of America (2007).

12. Gourich B, Belhaj Soulami M, Zoulalian A and Ziyad M, Simultaneous measurement of gas hold-up and mass transfer coefficient by tracer dynamic technique in "Emulsair" reactor whit an emulsion-venturi distributor. Chem Eng Sci 60:6414-6421 (2005)

13. Dursun $\mathrm{G}$ and Akosman C, Gas-liquid interfacial area and mass transfer coefficient in a co-current down flow contacting column. J Chem Technol Biot 81: 1859-1865 (2006)

14. Sotiriadis A, Thorpe R and Smith J, Bubble size and mass transfer characteristics of sparged downwards two-phase flow. Chem Eng Sci 60: 5917-5929 (2005). 
15. Fadavi A and Chisti Y, Gas-liquid mas transfer in a novel forced circulation loop reactor. Che Eng J 112:73-80 (2005).

16. Cachaza E, Díaz E, Montes F and Galán M, Analytical solution of the mass conservation equation in gas-liquid systems: Applicability to the evaluation of the volumetric mass transfer coefficient $\left(\mathrm{k}_{\mathrm{L}} \mathrm{a}\right)$. Industrial and Engineering Chemistry 47:4510-4522 (2008).

17. Evans G, Bin A and Machniewski P, Performance of confined plunging liquid jet bubble column as gas-liquid reactor. Chem Eng Sci 56: 1151-1157 (2001).

18. Cramers $\mathrm{P}$ and Beenackers $\mathrm{A}$, Influence of the ejector configuration, scale and the gas density on the mass transfer characterisitics of gas-liquid ejectors. Chem Eng $J$ 83:131-141 (2001).

19. Thomas B, Advances in chemical engineering, Volume 11, ed by Academic Press, New York, pp 93 (1981).

20. Gamisans X, Sarra M and Lafuente FJ, Fluid flow and pumping efficiency in an ejector-venturi scrubber. Chem Eng Process 43(2):127-136 (2004).

21. Letzel H, Schouten J, Krishna R and Bleek C, Gas holdup and mass transfer in bubble column reactors operated at elevated pressure. Chem Eng Sci 54:2237-2245 (1999).

22. Tobajas $\mathbf{M}$ and García-Calvo E, Comparison of experimental methods for determination of the volumetric mass transfer coefficient in fermentation processes. Heat mass transfer 36:201-207 (2000)

23. Painmanakul P and Hébrard G, Effect of different contaminants on the $\alpha$-factor: Local experimental method and modeling. Chemical Engineering Research and Design 86:1207-1215 (2008) 
24. Kennes $\mathrm{C}$ and Veiga MC, Bioreactors for waste gas treatment, ed by Kennes $\mathrm{C}$ and Veiga MC, Kluwer academic publishers, London, pp. 119 (2001). 
Table 1. Flows of air and water $\left(\mathrm{Q}_{\mathrm{G}}\right.$ and $\mathrm{Q}_{\mathrm{L}}$ respectively), and trickling liquid velocity (TLV) necessaries for the removal of 500 to $3000 \mathrm{ppm}_{\mathrm{v}}$ of $\mathrm{H}_{2} \mathrm{~S}$ in an industrial BTF with direct insertion in the recirculation line.

\begin{tabular}{|l|l|l|l|l|l|l|l|}
\hline Device & $\mathrm{H}_{2} \mathrm{~S}\left(\mathrm{ppm}_{\mathrm{v}}\right)$ & 500 & 1000 & 1500 & 2000 & 2500 & 3000 \\
\hline \multirow{5}{*}{ Jet-venturi } & $\mathrm{Q}_{\mathrm{G}}\left(\mathrm{m}^{3} \mathrm{~h}^{-1}\right)$ & 4.78 & 9.56 & 14.34 & 19.12 & 23.91 & 28.69 \\
\cline { 2 - 8 } & $\mathrm{Q}_{\mathrm{L}}\left(\mathrm{m}^{3} \mathrm{~h}^{-1}\right)$ & 4.29 & 8.57 & 12.86 & 17.14 & 21.43 & 25.72 \\
\cline { 2 - 8 } & $\mathrm{TLV}\left(\mathrm{m} \mathrm{h}^{-1}\right)$ & 3.23 & 6.46 & 9.69 & 12.92 & 16.15 & 19.38 \\
\hline \multirow{3}{*}{ Venturi ejector } & $\mathrm{Q}_{\mathrm{G}}\left(\mathrm{m}^{3} \mathrm{~h}^{-1}\right)$ & 7.97 & 15.95 & 23.92 & 31.89 & 39.87 & 47.84 \\
\cline { 2 - 8 } & $\mathrm{Q}_{\mathrm{L}}\left(\mathrm{m}^{3} \mathrm{~h}^{-1}\right)$ & 7.99 & 15.99 & 23.98 & 31.97 & 39.96 & 47.96 \\
\cline { 2 - 8 } & $\mathrm{TLV}\left(\mathrm{m} \mathrm{h}^{-1}\right)$ & 6.02 & 12.04 & 18.07 & 24.09 & 30.11 & 36.13 \\
\hline
\end{tabular}


Table 2. Flows of air and water $\left(\mathrm{Q}_{\mathrm{G}}\right.$ and $\mathrm{Q}_{\mathrm{L}}$ respectively) necessaries for the removal of 500 to $3000 \mathrm{ppm}_{\mathrm{v}}$ of $\mathrm{H}_{2} \mathrm{~S}$ in an industrial BTF with direct supply of air into the bottom of the BTF.

\begin{tabular}{|l|l|l|l|l|l|l|l|}
\hline Device & $\mathrm{H}_{2} \mathrm{~S}\left(\mathrm{ppm}_{\mathrm{v}}\right)$ & 500 & 1000 & 1500 & 2000 & 2500 & 3000 \\
\hline Jet-venturi & $\mathrm{Q}_{\mathrm{G}}\left(\mathrm{m}^{3} \mathrm{~h}^{-1}\right)$ & 4.78 & 9.56 & 14.34 & 19.12 & 23.91 & 28.69 \\
\cline { 2 - 8 } & $\mathrm{Q}_{\mathrm{L}}\left(\mathrm{m}^{3} \mathrm{~h}^{-1}\right)$ & 4.29 & 8.57 & 12.86 & 17.14 & 21.43 & 25.72 \\
\hline \multirow{2}{*}{$\begin{array}{l}\text { Venturi } \\
\text { ejector }\end{array}$} & $\mathrm{Q}_{\mathrm{G}}\left(\mathrm{m}^{3} \mathrm{~h}^{-1}\right)$ & 7.97 & 15.95 & 23.92 & 31.89 & 39.87 & 47.84 \\
\cline { 2 - 8 } & $\mathrm{Q}_{\mathrm{L}}\left(\mathrm{m}^{3} \mathrm{~h}^{-1}\right)$ & 7.99 & 15.99 & 23.98 & 31.97 & 39.96 & 47.96 \\
\hline
\end{tabular}


Figure captions:

Figure 1: Schematic of the mass transfer pilot plant. PFV: Pump with Frequency Variator, ET: Expansion Tank, DIF: Diffuser, SV: Separation Vessel, VE / JVE : Venturi Ejector / Jet-Venturi, FI3: Water Flowmeter, FI1-FI2: Gas Flowmeters, VL: Optical Level, DO1-DO2: DO Sensors, DT: Down flow tube, PTP: Pressure transducer and PSV: Pressure switch and safety valve. Discontinuous line indicates electric lines, and continuous process lines.

Figure 2(a): Raw experimental data and simulations for the venturi ejector for $\mathrm{Q}_{\mathrm{L}}=6.00$ $\mathrm{x} 10^{-4} \mathrm{~m}^{3} \mathrm{~s}^{-1}$ and $\mathrm{Q}_{\mathrm{G}}=6.00 \times 10^{-4} \mathrm{~m}^{3} \mathrm{~s}^{-1}$

Figure 2(b): Raw experimental data and simulations for the jet-venturi for $Q_{L}=5.67 \mathrm{x}$ $10^{-4} \mathrm{~m}^{3} \mathrm{~s}^{-1}$ and $\mathrm{Q}_{\mathrm{G}}=6.5 \times 10^{-4} \mathrm{~m}^{3} \mathrm{~s}^{-1}$

Figure 3: Comparison of SAE for the diffuser, venturi ejector and jet-venturi.

Figure 4: Effect of pressure inside the tank on the SOTR for the venturi ejector and the jet-venturi.

Figure 5(a): Effect of sulfate content on the SOTR for (a) the venturi ejector and (b) for the jet venturi 
Table captions:

Table 1. Flows of air and water $\left(\mathrm{Q}_{\mathrm{G}}\right.$ and $\mathrm{Q}_{\mathrm{L}}$ respectively), and trickling liquid velocity (TLV) necessaries for the removal of 500 to $3000 \mathrm{ppm}$ of $\mathrm{H}_{2} \mathrm{~S}$ in an industrial BTF with direct insertion in the recirculation line.

Table 2. Flows of air and water $\left(\mathrm{Q}_{\mathrm{G}}\right.$ and $\mathrm{Q}_{\mathrm{L}}$ respectively) necessaries for the removal of 500 to 3000 ppm of $\mathrm{H}_{2} \mathrm{~S}$ in an industrial BTF with direct supply of air into the bottom of the BTF. 
Figure 1. Created with autoCAD2009

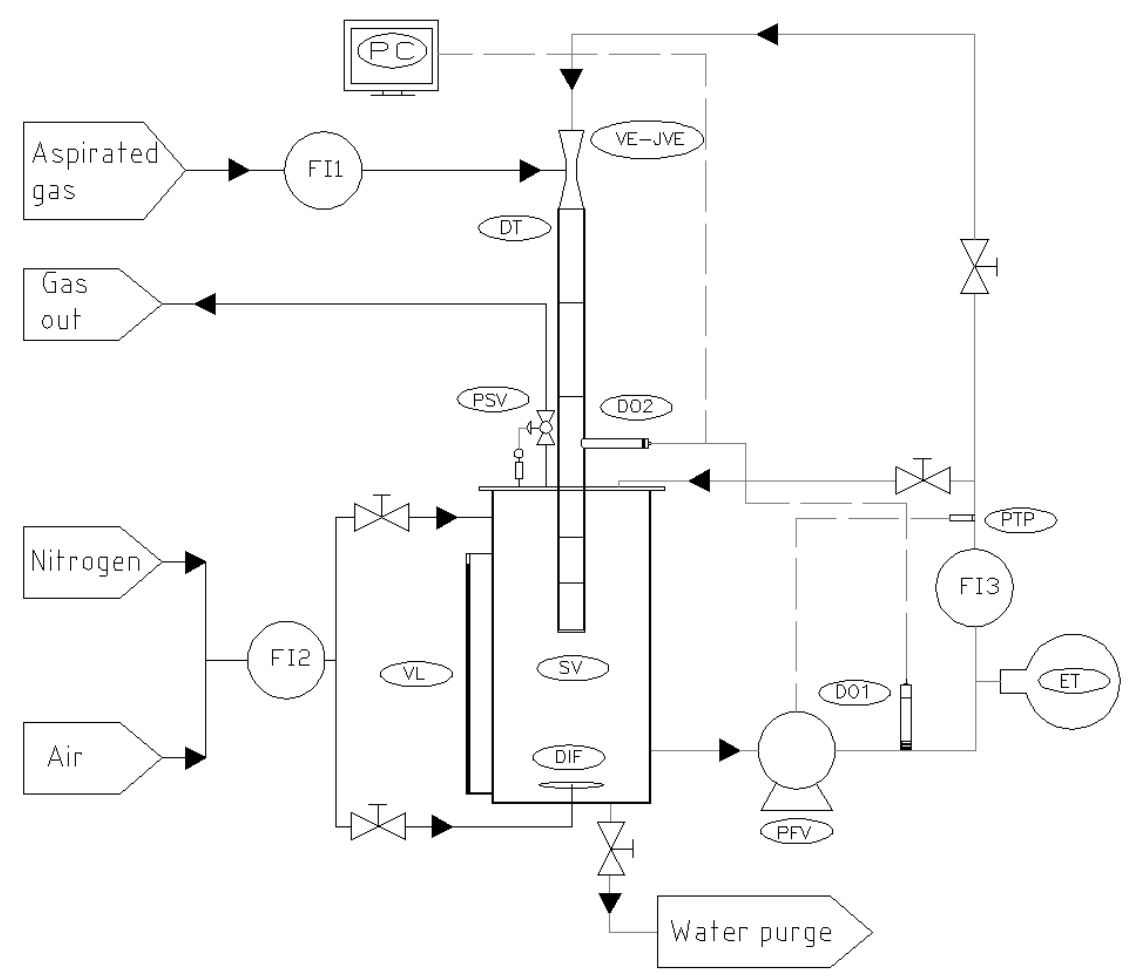


Figure 2(a). Created with Matlab R2009b

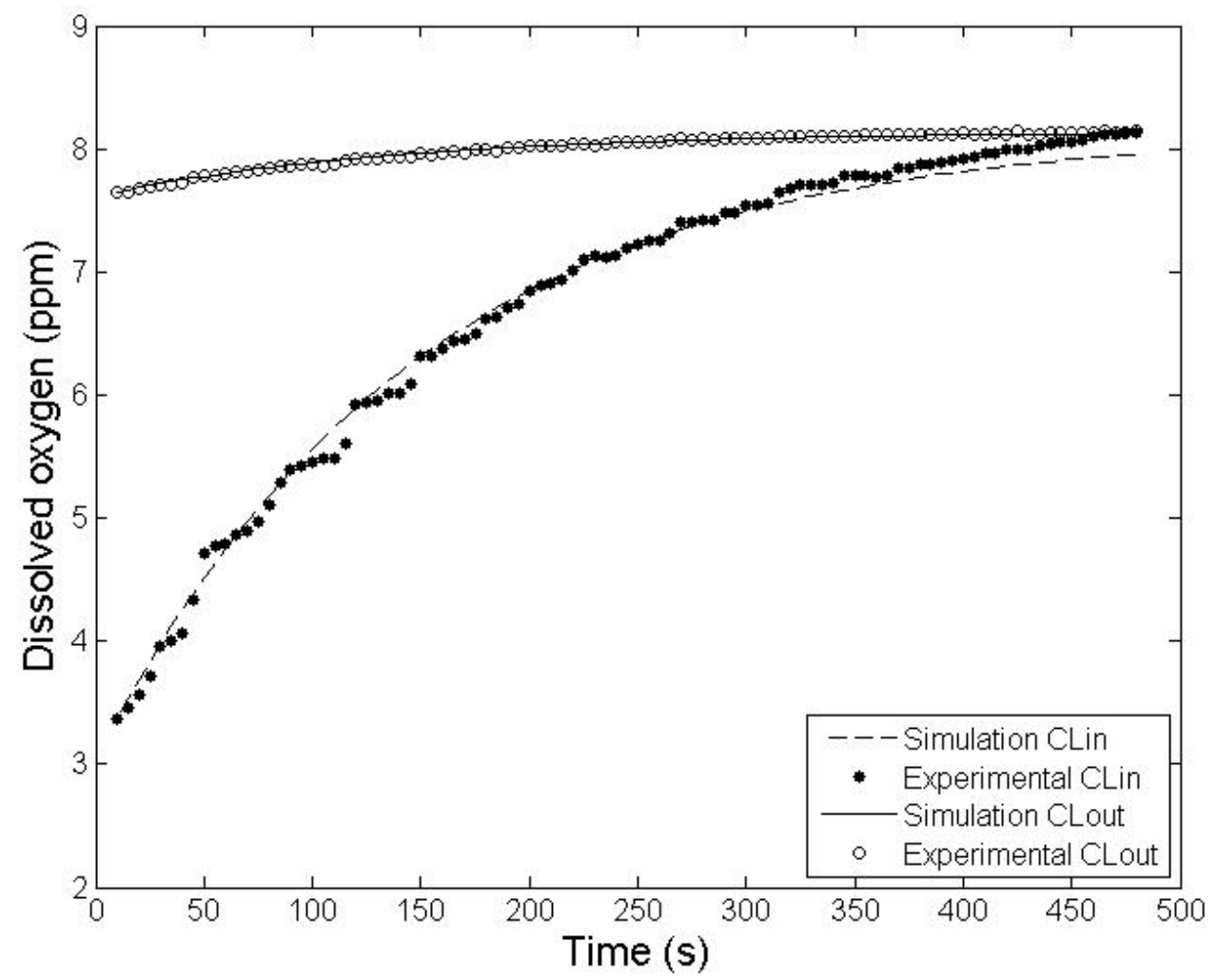


Figure 2(b). Created with Matlab R2009b

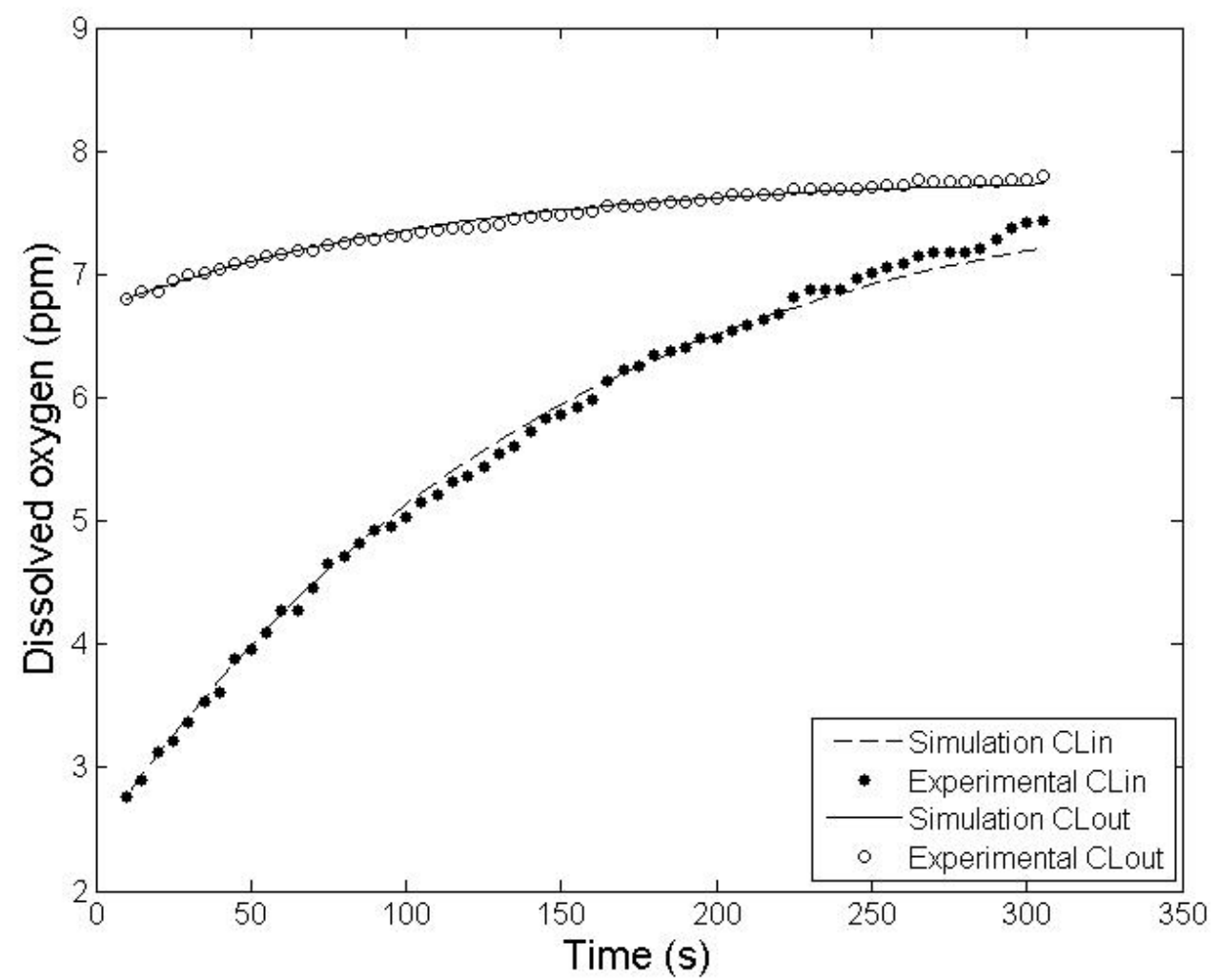


Figure 3. Created with Sigmaplot 12.0

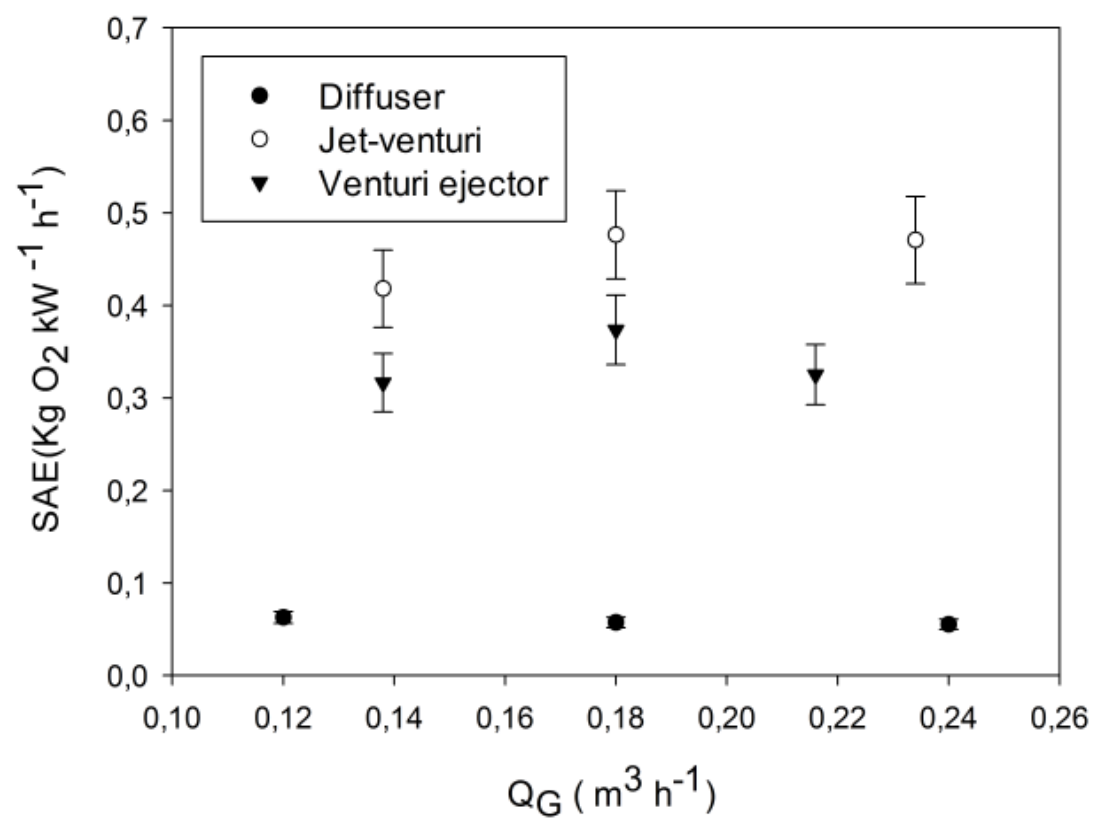


Figure

4

Created

with

Sigmaplot

12.0

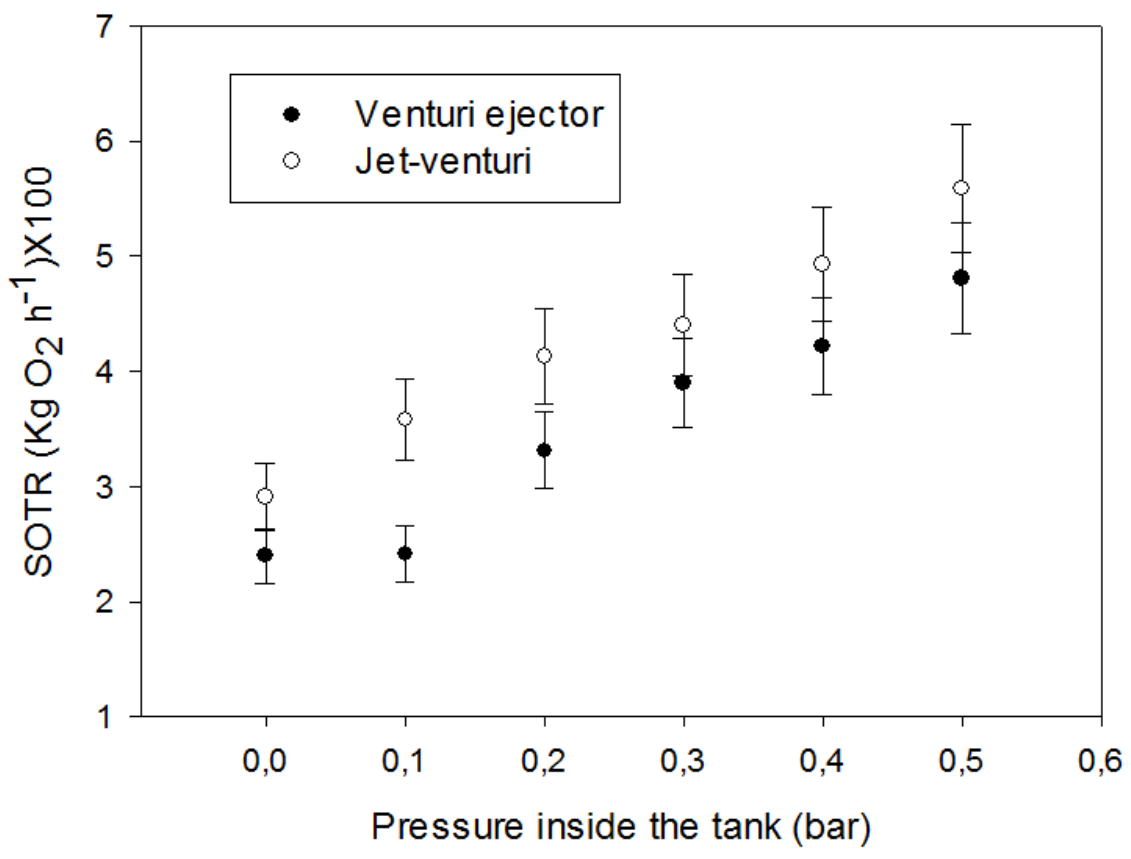


Figure 5(a). Created with Sigmaplot 12.0

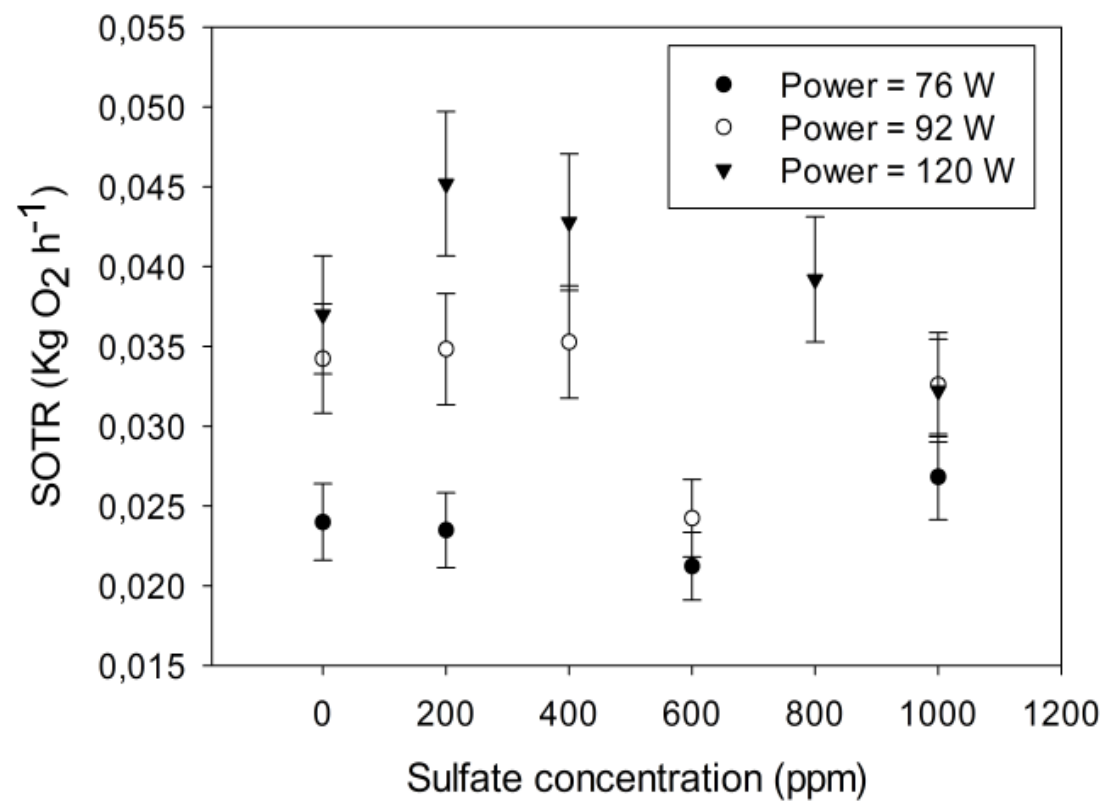


Figure 5(b). Created with Sigmaplot 12.0

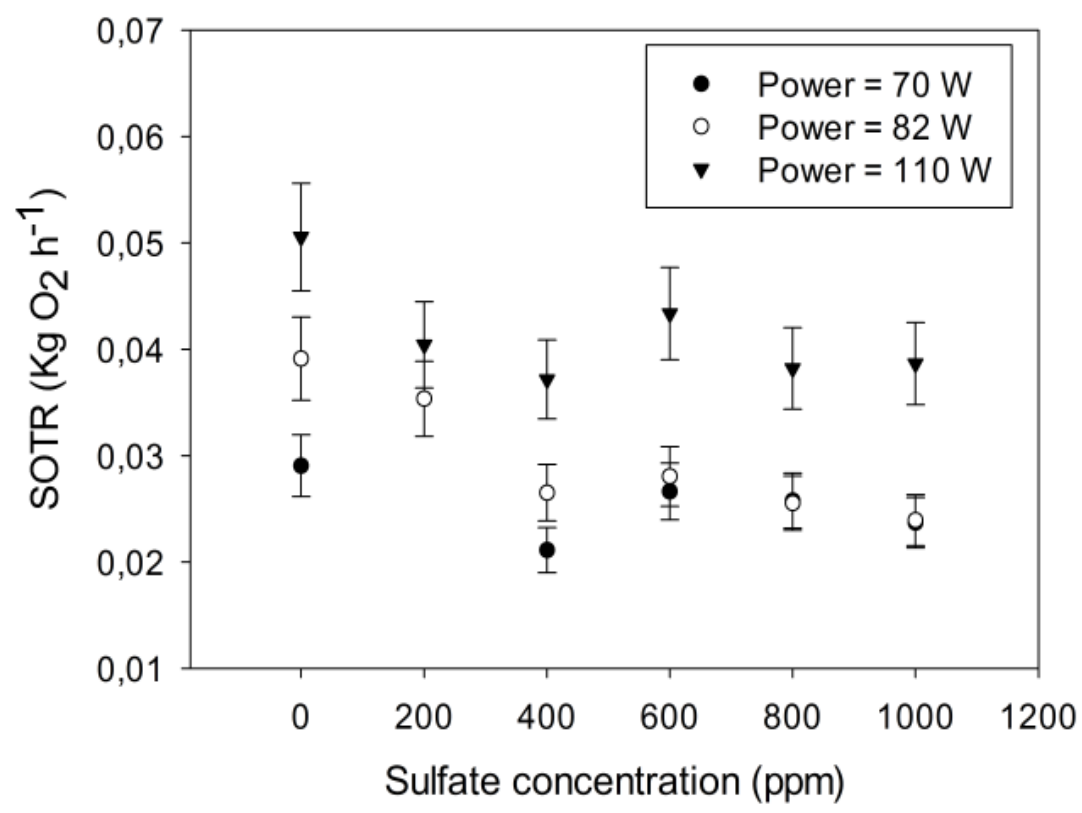

